\title{
NEUROENDOCRINOLOGY
}

\section{Cholesterol metabolites regulate motor neuron function}

Cholestenoic acids-intermediates in the metabolism of cholesterol to bile acids —differentially regulate motor neuron survival in mice and possibly in humans, show Theofilopoulos et al. in The Journal of Clinical Investigation. The findings have potential implications for the diagnosis and treatment of motor neuron disorders.

Cholesterol is a major constituent of the human brain and defects in its metabolism can lead to disorders of the central nervous system, including motor neuron degeneration. A key mechanism in developmental and adult regulation of neuronal function involves the activation of nuclear receptors. "Previous work from the Karolinska Institute, Sweden, has shown that the liver $\mathrm{X}$ receptor (LXR) class of nuclear receptors are important in the

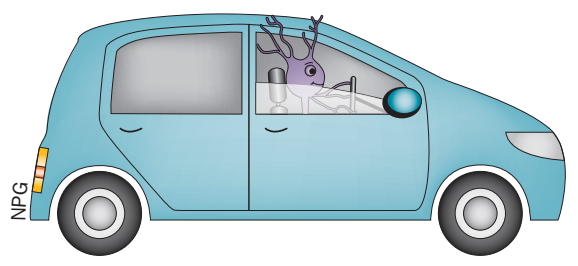

development of motor neurons and for their maintenance in adult life; however, until now, no endogenous ligand capable of regulating the survival of motor neurons has been identified," explains study investigator William Griffiths.

The researchers used liquid chromatography-electrospray ionizationmass spectrometry to analyse the cholestenoic acid profile of cerebrospinal fluid (CSF) and plasma isolated from patients with two neurological diseases associated with motor neuron degeneration: cerebrotendinous xanthomatosis (CTX); and hereditary spastic paresis type 5 (SPG5).

Patients with CTX or SPG5 had reduced CSF and plasma levels of $3 \beta, 7 \alpha$-dihydroxycholest-5-en26-oic acid ( $3 \beta, 7 \alpha$-diHCA); patients with SPG5 additionally had elevated levels of $3 \beta$-hydroxycholest-5-en26-oic acid (3 $\beta$-HCA). Functional analyses of these cholestenoic acids in mice revealed that $3 \beta, 7 \alpha$-diHCA promoted motor neuron survival in an LXR-dependent manner, whereas $3 \beta$-HCA induced motor neuron loss. Moreover, administration of $3 \beta, 7 \alpha$-diHCA blocked the loss of motor neurons induced by $3 \beta$-HCA in developing mouse brains in vivo. "Our results indicate that specific cholestenoic acids selectively work on motor neurons via LXR to regulate the balance between survival and death," states Griffiths.

The researchers plan to investigate the neuroprotective potential of $3 \beta, 7 \alpha$-diHCA, and inhibitors of enzymes involved in its metabolic conversion, for treating patients with motor neuron disorders, and whether an abnormal cholestenoic acid profile can be used as a biomarker for diagnosing motor neuron degeneration. Considering the paucity of approved therapeutics for treating motor neuron disease, further studies on cholestenoic acids are warranted.

David Holmes

Original article Theofilopoulos, S. et al. Cholestenoic acids regulate motor neuron survival via liver $\mathrm{X}$ receptors. J. Clin. Invest. doi:10.1172/JCl68506 